\title{
The Epidemiological and Economic Impact of a Cell- Based Quadrivalent Influenza Vaccine in an Adult Population in the US: A Dynamic Modeling Approach
}

\author{
Van Hung Nguyen ( $\nabla$ vhnguyen@vhnconsulting.com ) \\ VHN consulting https://orcid.org/0000-0002-0371-0193 \\ Yvonne Hilsky \\ Seqirus Vaccines Ltd \\ Joaquin Mould-Quevedo \\ Seqirus Vaccines Ltd
}

\section{Research article}

Keywords: Influenza, cost-effectiveness, Quadrivalent Influenza Vaccine, cell-based influenza vaccine, US

Posted Date: September 9th, 2020

DOl: https://doi.org/10.21203/rs.3.rs-27872/v2

License: (c) (i) This work is licensed under a Creative Commons Attribution 4.0 International License.

Read Full License 


\section{Abstract}

Background: Mutations of the $\mathrm{H} 3 \mathrm{~N} 2$ vaccine strain during the egg-based vaccine manufacturing process seem to partly explain the suboptimal effectiveness of traditional seasonal influenza vaccine. Cell-based influenza vaccines avoid such egg-adaptation, thereby improving antigenic match and vaccine effectiveness. The objective of this study was to evaluate the public health and economic impact of a cell-based quadrivalent influenza vaccine (QIVc) in adult population (18-64 years) compared to the standard egg-based quadrivalent influenza vaccine (QIVe), in the US.

Methods: The impact of QIVc over QIVe in terms of public health and costs outcomes was estimated using a dynamic SEIR transmission model. The model is age-structured and accounts for 4 circulating influenza strains (A/H1 N1pdm9, A/H3N2, B(Victoria), and B(Yamagata)). It was calibrated on US attack rate and strain circulation for the influenza seasons 2013-2018. US specific absolute vaccine effectiveness for QIVe, specific hospitalization rate, mortality rate, Quality-Adjusted Life Years (QALYs) and costs were extracted from published literature. Relative vaccine effectiveness of QIVc over QIVe for subjects 18-64 years of age was obtained from a US retrospective cohort study. Robustness of the results was assessed in univariate and probabilistic sensitivity analyses.

Results: Switching from QIVe to QIVc in the 18-64 year-old population may prevent 5.7 M symptomatic cases, 1.8 M outpatient visits; $50 \mathrm{~K}$ hospitalizations and 5,453 deaths annually. The switch could save $128 \mathrm{~K}$ QALYs and US\$ 845M in direct costs, resulting in a cost-saving alternative in a 3-year time horizon analysis. Probabilistic sensitivity analyses confirmed the robustness of the cost-saving result.

Conclusions: The analysis shows that QIVc is expected to prevent a substantial number of hospitalizations and deaths, and would result in substantial savings in health care costs.

\section{Background}

With an estimated average of 410,000 deaths yearly (1), the burden of influenza infection still weighs heavily on the world population, despite years of improvement in worldwide immunization policies. In the US only, the US CDC estimates that influenza infections have caused the sickness of $35.5 \mathrm{M}$ individuals, 16.5M outpatient visits, 490,600 hospitalizations and 34,200 deaths during the 2018-2019 winter season (2). Vaccination against influenza is generally considered the most effective way to prevent influenza infection and its consequences. However, the complexities of influenza viruses, evading the human immune response with antigenic drift and shift phenomena, competing with a diversity of other strains for dominant circulation, seriously challenge, every year, the effectiveness of influenza immunization campaigns. Recent years have shown advances in influenza vaccination policies, with the adoption by some (mainly high developed) countries of quadrivalent influenza vaccines, containing antigens for two $B$ lineages, instead of a single one, and relatively new adjuvanted and high-dose formulations. Still, conventional egg-based influenza vaccines seem to offer a suboptimal protection during influenza seasons dominated by influenza $A(H 3 N 2)$ circulation. A recent meta-analysis measured a $35 \%$ vaccine 
effectiveness (VE) against $A(H 3 N 2)$ versus $54-73 \%$ for other strains, for working-age adults, vaccinated with egg-based influenza vaccines (3). This poor protection seems to be partially explained by issues related to the production of egg-based vaccines. Mutations of influenza virus strains during the isolation and propagation steps of egg-based vaccine production seem to be leading to a mismatch between the influenza vaccine strain and the circulating one $(4,5)$. Solving this issue may rely on the use of mammalian cells rather than chicken eggs during vaccine virus isolation and propagation. This cell based production process has demonstrated an improved antigenic similarity between the original candidate vaccine virus and the circulating wild-type influenza virus strains, which may translate into an increased VE for new cell-based quadrivalent influenza vaccines (QIVc) $(6,7)$.

The assessment of the public health and economic impact of new influenza immunization policies is routinely done for most countries. Despite WHO recommendations for the assessment of vaccination strategies(8), most of these studies are performed using a simple static epidemiological model(9), unable to grasp the indirect effects of vaccination (herd effects). While the potential impact of improved influenza vaccine effectiveness is generally acknowledged for the pediatric population (10), its impact on the adult population remains unclear. The purpose of the present analysis is to evaluate the costeffectiveness of QIVc in adults (18-64 years) compared to conventional egg-based quadrivalent influenza vaccines (QIVe) using a dynamic influenza transmission model able to account for the indirect effect of vaccination.

\section{Methods}

\section{Epidemiology and vaccine effectiveness}

Influenza incidence estimates were extracted from CDC reports for 5 influenza seasons from 2013 to 2018 (Table S1) (11), and combined with WHO FluNet (12) virological data to obtain yearly incidence per strain for the US. The model assumed $66 \%$ of people infected with influenza virus were symptomatic (13), that they had an incubation period of 0.8 days, and remained infectious for 1.8 days $(14,15)$.

We used strain specific QIVe VE estimates obtained by Rolfes et al. for the influenza season 2017-2018 (16) assuming VE against $A(H 3 N 2)$ was a mismatch between the vaccine and the circulating strain. To estimate VE conferred by QIVc, we extracted results from a recent study estimating the relative VE (rVE) of QIVc compared to QIVe from electronic medical records, where patients were matched by propensity score for the influenza season 2017-2018 (17), i.e. the same influenza season as in Rolfes et al. This study estimated an overall rVE of $19.3 \%$ (95\% $\mathrm{Cl}[9.5 \% ; 28.0 \%])$ for QIVc compared to QIVe, and ageadjusted rVE for adult population (Table 1). In order to compute the specific QIVc increased effectiveness against $\mathrm{A}(\mathrm{H} 3 \mathrm{~N} 2)$ (in 2017-2018, only $\mathrm{A}(\mathrm{H} 3 \mathrm{~N} 2)$ antigens are cell-based), we used the total rVE estimated on a US cohort comparing QIVc and QIVe for the same influenza season, and then, we recomputed, the QIVc VE against $A(H 3 N 2)$ using the following equations: see equations 1, 2, and 3 in the supplementary files. 
A four strains compartmental transmission model was developed to provide estimates of the epidemiological impact of the switch from QIVe to QIVc. The model is a classic SEIR model where the population can be either Susceptible to infection with Influenza strain $I\left(S_{i}\right)$, Exposed to the strain $\left(E_{i}\right)$, Infected and infectious $\left(I_{i}\right)$ or Recovered from infection $\left(R_{i}\right)$ (Figure 1$)$. The vaccinated population could still be infected and contribute to the infection dynamic but with a reduced probability corresponding to the vaccine effectiveness against the given influenza strain. The model simulates independently the epidemiological dynamics of $A(H 1 N 1) p d m 09, A(H 3 N 2), B /$ Victoria and $B /$ Yamagata for a given influenza season. The model is structured by age-group (6-23mo, 2-4yo, 5-12yo, 13-17yo, 18-49yo, 50-64yo, and more than 65yo) and uses a contact matrix to account for assortative rate of contacts between agegroups. In our analysis, we used in our base case analysis the matrix from Mossong et al.(18) and have conducted sensitivity analysis using Zagenhi et al.(19). Both matrices have provided qualitatively similar results. Probabilities of influenza transmission per influenza strain are estimated for each influenza season to match US reported strain specific attack rate (2013 to 2018 influenza seasons). We assume a pre-immunity of a third of the population based on estimations from Baguelin et al. (20). The estimation process uses the non-linear Nelder-Mead simplex algorithm(21) to maximize a likelihood function.

The economic model is based on a decision tree model published in De Boer et al. (22) whose inputs are given in Table S3. We used the same methodology and same health outcomes computed on the whole population. The number of cases per age-group estimated by the epidemiological model, shown in Table 2 , are taken as inputs of the economic model. Probabilities of general practitioner visit, hospitalization, and death are applied on the number of cases attributed to a high or low risk group, and then translated into public health outcomes and costs. The economic analysis is performed from a societal perspective without taking into account productivity loss due to death. We consider a willingness to pay per QALY threshold of US\$50 000 to consider a strategy as cost-effective (23).

\section{Economic data}

Disease costs and QALYs were extracted from a recent influenza health economic analysis performed in the US context (22). Cost of a work day for the pediatric population are assumed to be related to parental work loss. Vaccine cost for QIVe and QIVc were set at \$17.22 and \$24.22 respectively(24). We do not consider administration costs since they are assumed to be the same across the different vaccination strategy (no difference in vaccination coverage). Details of the costs per age-groups are given in Table S3.

\section{Scenarios}

As a reference strategy, we assume that the US population is vaccinated with conventional QIVe for those aged under 65 years of age and TIV HD for those aged 65 years and above. Then we compare this strategy to a scenario where QIVe is replaced by QIVc for people aged 18-64 years, other age-groups keeping their baseline vaccination. For both scenarios, we use age-based vaccination coverage rate documented by $\operatorname{CDC}(25)$, in particular we consider that $34.9 \%$ of people aged 18 to $49 y 0,47.30 \%$ of 
people aged 50 to $64 \mathrm{yo}$, and $68.10 \%$ of people older than 65 years are vaccinated against influenza (Table S2). In this base case comparison, we consider that a seasonal mismatch between the QIVe $\mathrm{A}(\mathrm{H} 3 \mathrm{~N} 2)$ strain and the circulating strain due to egg adaptation occurred during the last 3 years out of 5 years in the analysis scope (26). As a sensitivity analysis we also assessed the impact of QIVc when the mismatch due to egg adaptation occurred over a varying number of years from 1 to 5 years, and randomly picking the influenza season concerned by the mismatch.

We also performed a stochastic probabilistic sensitivity analysis in order to assess the robustness of our results regarding uncertainties in vaccine effectiveness, economic inputs (primary care and hospitalization costs), probability of outcomes, listed in Table S3 and Table S4, with their probability distributions. In this analysis, 1,000 sets of the above-mentioned parameters are randomly drawn from distributions indicated in the Table S2 and Table S3. Clinical and economic results are averaged over the 5 influenza seasons.

\section{Results}

In our base case scenario of 3 seasonal $\mathrm{A}(\mathrm{H} 3 \mathrm{~N} 2)$ mismatches due to egg adaptation, our analysis shows that using QIVc instead of QIVe in the 18-64yo population would have prevented 5.7M cases of influenza, 1.8M GP visits, almost 50K hospitalizations and more than 5,400 deaths. In total, QIVc would have saved US\$845M in direct costs and saved 128K QALYs. Hence, the switch from QIVe to QIVc in the adult population would be a cost saving strategy (Table 2).

Over 5 influenza seasons of $A(H 3 N 2)$ mismatch, using QIVc instead of QIVe in the 18-64yo population would have a substantial effect but irregular effect, depending on the influenza season, depending on $A(H 3 N 2)$ seasonal circulation (Figure 2). Hence, the choice of the influenza season was also randomly varied in the probabilistic analysis to assess the uncertainty related to the epidemiological context. Varying the number of influenza seasons with an A(H3N2) mismatch of egg-based vaccines between 1 and 5 years still show that QIVc would be cost-saving or very cost effective (Table 3). Probabilistic sensitivity analysis confirms that $95 \%$ of the 1,000 simulations gave a cost-saving result (Figure 3 ).

\section{Discussion}

This analysis emphasizes the potentially major public health gain which could be achieved through the use of QIVc. Several studies have highlighted the suboptimal VE of egg-based vaccines against some strains of $A(H 3 N 2)(6,27)$. While the link between VE reduction and egg-related strain mutations is still poorly understood (28) and remains to be fully investigated, recent studies, in different populations, tend to confirm the clinical benefits (7) of QIVc over QIVe regarding A(H3N2) vaccine strain mismatch. Assessing the strain-specific rVE of QIVc compared to QIVe remains a difficult challenge as eggadaptation phenomena, while most common on $A(H 3 N 2)$, may also occur on $B$ lineages. However, we used QIVc rVE estimated during the 2017-2018 influenza season, when only the A(H3N2) component of QIVc had been grown in cells and we derived our strain specific VE estimations only from this influenza 
season. Our analysis relies on retrospective studies $(7,17)$ performed during the 2017-2018 influenza season when $A(H 3 N 2)$ represented $66 \%$ of the influenza positive samples (12). By construction, these analyses were only able to assess non-strain specific QIVc rVE compared to QIVe, and we had to estimate QIVc strain specific VE, assuming that the increased total VE was only linked to an increase of VE against $\mathrm{A}(\mathrm{H} 3 \mathrm{~N} 2)$. Also, variations in QIVc rVE are likely to occur due to the changing distribution patterns of circulating influenza strains. Namely, influenza seasons with a highly dominant A(H1N1)pdm09 circulation (2015-2016) will see a low benefit to QIVc compared to QIVe, while others, like 2014-2015, 2016-2017, or 2017-2018, may see a significant one. Hence, analysis on multiple influenza seasons is necessary in order to fully assess the potential "averaged" impact of cell-based vaccines across various realistic epidemiological contexts.

We chose to consider as a base case scenario that a mismatch occurred between the $A(H 3 N 2)$ circulating strain and the egg-based vaccine strain during three influenza seasons. We considered this choice a median scenario between a systematic yearly mismatch and no mismatch at all. In addition, this assumption has a limited impact on our results since 1) we consider the observed influenza strain distribution, and 2) we have analyzed situation when the number of mismatched years was varied from 1 to 5 years and reached qualitatively similar results. In addition, our results are consistent with previous health-economic analysis of QIVc in Europe (UK, Spain, Italy, Germany) (29-31), where QIVc has been shown to be either cost-saving or highly cost-effective.

Our analysis uses a 4-strain SEIR compartmental model with an age-structure. This kind of approach, previously used in several similar analysis $(9,32-34)$, is key to capture the potential indirect effects of influenza vaccination, accounting for prevented chains of transmission. However, it relies on assumptions regarding age-related contact rate which may be difficult to measure for specific countries. Despite its advantages, our approach suffers from limitations inherent to any modeling exercise. In absence of better estimates, our transmission model assumes that $30 \%$ of the population benefits from remaining immunity against influenza based on a British modeling study(14). In addition, uncertainties regarding surveillance-based influenza incidence estimates, or influenza strains circulations in the US will directly impact the epidemiological dynamics reproduced by the model.

Finally, our analysis shows the potential public health benefits of the use of QIVc in the 18-64yo US population during the 2013-2018 period. Results obtained retrospectively may be different from what may be achieved in the future as influenza strain circulation is currently challenging to predict. In particular, given the potential expected benefits, and on-going clinical trials, it is likely that QIVc would be also recommended for the pediatric population, which would certainly reinforce its impact.

\section{Conclusions}

Estimated public health benefits related to the use of QIVc in the US adult population, indicate that the use of QIVc could be clinically superior and cost-saving compared to the current vaccination practice 
(QIVe). Sensitivity analyses on vaccine effectiveness, costs and number of influenza seasons of eggbased strain mismatch show the robustness of QIVc cost-effectiveness.

\section{Declarations}

Ethics approval and consent to participate

Not applicable

Consent to publish

Not applicable

Availability of data and materials

All data generated or analysed during this study are included in this published article.

Competing Interests

J.M.Q. and Y.H. are employees of Seqirus USA Inc. V.H.N. was contracted by Seqirus and received fees for conducting the study.

Funding

The study was funded by Seqirus. The funder had no role in the study design, data collection, analysis, decision to publish or preparation of the manuscript.

Authors' contribution

V.H.N., J.M.Q. and Y.H. contributed to the design and implementation of the research, to the analysis of the results and to the writing of the manuscript. All authors have read and approved the manuscript.

\section{Abbreviations}

QIVe: egg-based Quadrivalent Influenza Vaccine

QIVc: cell-based Quadrivalent Influenza Vaccine

VE: Vaccine Effectiveness

RVE: Relative Vaccine Effectiveness

\section{References}


1. Iuliano AD, Roguski KM, Chang HH, Muscatello DJ, Palekar R, Tempia S, et al. Estimates of global seasonal influenza-associated respiratory mortality: a modelling study. Lancet. 2018;391(10127):1285-300.

2. Center for Disease Control and Prevention. Estimated Influenza IIInesses, Medical visits, Hospitalizations, and Deaths in the United States - 2018-2019 influenza season. 2019.

3. Belongia EA, Simpson MD, King JP, Sundaram ME, Kelley NS, Osterholm MT, et al. Variable influenza vaccine effectiveness by subtype: a systematic review and meta-analysis of test-negative design studies. Lancet Infect Dis. 2016 Aug 1;16(8):942-51.

4. Wu NC, Zost SJ, Thompson AJ, Oyen D, Nycholat CM, McBride R, et al. A structural explanation for the low effectiveness of the seasonal influenza H3N2 vaccine. PLoS Pathog. 2017 Oct 1;13(10).

5. Zost SJ, Parkhouse K, Gumina ME, Kim K, Perez SD, Wilson PC, et al. Contemporary H3N2 influenza viruses have a glycosylation site that alters binding of antibodies elicited by egg-adapted vaccine strains. Proc Natl Acad Sci U S A. 2017 Nov 21;114(47):12578-83.

6. DeMarcus L, Shoubaki L, Federinko S. Comparing influenza vaccine effectiveness between cellderived and egg-derived vaccines, 2017-2018 influenza season. Vaccine [Internet]. 2019;37(30):4015-21. Available from: https://doi.org/10.1016/j.vaccine.2019.06.004

7. Izurieta HS, Chillarige Y, Kelman J, Wei Y, Lu Y, Xu W, et al. Relative Effectiveness of Cell-Cultured and Egg-Based Influenza Vaccines among Elderly Persons in the United States, 2017-2018. J Infect Dis. 2019;220(8):1255-64.

8. World Health Organization (WHO). Guidance on the economic evaluation of influenza vaccination. 2016.

9. de Boer PT, van Maanen BM, Damm O, Ultsch B, Dolk FCK, Crépey P, et al. A systematic review of the health economic consequences of quadrivalent influenza vaccination. Expert Rev Pharmacoeconomics Outcomes Res. 2017;17(3).

10. Fleming DM, Elliot AJ. Health benefits, risks, and cost-effectiveness of influenza vaccination in children. Pediatr Infect Dis J. 2008/11/26. 2008;27(11 Suppl):S154-8.

11. Centers for Disease Control and Prevention. Weekly US Influenza report, United States, 2013-18 influenza seasons.

12. WHO. FluNet, Global Influenza Surveillance and Response System [Internet]. [cited 2019 Jun 2]. Available from: www.who.int/flunet

13. Carrat F, Vergu E, Ferguson NM, Lemaitre M, Leach S, Valleron A. Meta-Analysis Time Lines of Infection and Disease in Human Influenza: A Review of Volunteer Challenge Studies. Am J Epidemiol. 2008;167(7):775-85.

14. Baguelin M, Flasche S, Camacho A, Demiris N, Miller E, Edmunds WJ. Assessing optimal target populations for influenza vaccination programmes: an evidence synthesis and modelling study. PLoS Med [Internet]. 2013 Oct [cited 2014 Feb 25];10(10):e1001527. Available from: http://www.pubmedcentral.nih.gov/articlerender.fcgi? artid=3793005\&tool=pmcentrez\&rendertype=abstract 
15. Ferguson NM, Cummings D a T, Cauchemez S, Fraser C, Riley S, Meeyai A, et al. Strategies for containing an emerging influenza pandemic in Southeast Asia. Nature. 2005 Sep;437(7056):209-14.

16. Rolfes MA, Flannery B, Chung JR, O'Halloran A, Garg S, Belongia EA, et al. Effects of Influenza Vaccination in the United States during the 2017-2018 Influenza Season. Clin Infect Dis. 2019;69(11):1845-53.

17. Boikos C, Sylvester G, Sampalis J, Mansi J. Effectiveness of the Cell Culture- and Egg-Derived, Seasonal Influenza Vaccine during the 2017-2018 Northern Hemisphere Influenza Season. submitted. 2019;

18. Mossong J, Hens N, Jit M, Beutels P, Auranen K, Mikolajczyk R, et al. Social contacts and mixing patterns relevant to the spread of infectious diseases. PLoS Med. 2008/03/28. 2008 Mar;5(3):e74.

19. Zagheni E, Billari FC, Manfredi P, Melegaro A, Mossong J, Edmunds WJ. Using Time-Use Data to Parameterize Models for the Spread of Close-Contact Infectious Diseases. Am J Epidemiol [Internet]. 2008 Sep 18 [cited 2017 Apr 14];168(9):1082-90. Available from:

https://academic.oup.com/aje/article-lookup/doi/10.1093/aje/kwn220

20. Baguelin M, Camacho A, Flasche S, Edmunds WJ. Extending the elderly- and risk-group programme of vaccination against seasonal influenza in England and Wales: a cost-effectiveness study. BMC Med [Internet]. 2015;13(1):236. Available from: http://dx.doi.org/10.1186/s12916-015-0452y\%5Cnhttp://www.biomedcentral.com/1741-7015/13/236

21. Nelder JA, Mead R. A Simplex Method for Function Minimization. Comput J. 1965 Jan 1;7(4):30813.

22. de Boer PT, Crépey P, Pitman RJ, Macabeo B, Chit A, Postma MJ. Cost-Effectiveness of Quadrivalent versus Trivalent Influenza Vaccine in the United States. Value Heal [Internet]. 2016;1-12. Available from: http://linkinghub.elsevier.com/retrieve/pii/S1098301516305034

23. Grosse SD. Assessing cost-effectiveness in healthcare: History of the $\$ 50,000$ per QALY threshold. Expert Rev Pharmacoeconomics Outcomes Res. 2008;8(2):165-78.

24. CDC. Archived CDC Vaccine Price List as of July 1, 2019 [Internet]. Available from: https://www.cdc.gov/vaccines/programs/vfc/awardees/vaccine-management/price-list/2019/201907-01.html

25. Centers for Disease Control and Prevention. Flu Vaccination Coverage, United States, 2018-19 Influenza Season [Internet]. Available from: https://www.cdc.gov/flu/fluvaxview/coverage1819estimates.htm

26. Rajaram S, Van Boxmeer J, Leav B, Suphaphiphat P, Iheanacho I, Kistler K. Retrospective Evaluation of Mismatch From Egg-Based Isolation of Influenza Strains Compared With Cell-Based Isolation and the Possible Implications for Vaccine Effectiveness. 2018;5(Suppl 1):S69.

27. Bruxvoort KJ, Luo Y, Ackerson B, Tanenbaum HC, Sy LS, Gandhi A, et al. Comparison of vaccine effectiveness against influenza hospitalization of cell-based and egg-based influenza vaccines, 2017-2018. Vaccine [Internet]. 2019;37(39):5807-11. Available from: https://doi.org/10.1016/j.vaccine.2019.08.024 
28. Belongia EA, McLean HQ. Influenza Vaccine Effectiveness: Defining the H3N2 Problem. Clin Infect Dis. 2019;69(10):1817-23.

29. Nguyen V, Ruiz-Aragón J, Nasiri M, Mould-Quevedo J, Rajaram S. The economic advantages of a cellbased quadrivalent influenza vaccine in the adult population in Europe. The Cost-Effectiveness Evidence in United Kingdom and Spain. Options Control Influ. 2019;

30. Rizzo C, Capri S, Merler S. Valutazione economica dell'intro- duzione del nuovo vaccino antinfluenzale quadrivalente da coltura cellulare (Flucelvax® Tetra) nel contesto di cura italiano. Ital J Public Health. 2019;8(5).

31. Cai R, Eichner M, Mould-Quevedo J, Lamotte M. Kosteneffektivität des zellbasierten quadrivalenten Influenza-Impfstoffs (QIVc) im Vergleich zum standardmäßigen quadrivalenten Impfstoff auf Eibasis (QIVe) in Deutschland. Ergebnisse einer dynamischen Transmissionsmodellierung. In: Wettbewerb und Medizinische Innovation. Wuppertal; 2020.

32. Baguelin M, Jit M, Miller E, Edmunds WJ. Health and economic impact of the seasonal influenza vaccination programme in England. Vaccine [Internet]. 2012 May 14 [cited 2014 Feb 25];30(23):3459-62. Available from: http://www.ncbi.nlm.nih.gov/pubmed/22446636

33. Thommes EW, Chit A, Meier GC, Bauch CT. Examining Ontario's universal influenza immunization program with a multi-strain dynamic model. Vaccine. 2014 Sep 3;32(39):5098-117.

34. Crépey P, Boer PT De, Postma MJ, Pitman R. Retrospective public health impact of a quadrivalent influenza vaccine in the United States. Influenza Other Respi Viruses. 2015;9(S1):39-46.

\section{Tables}

Table 1: Vaccine effectiveness (VE) for QIVe and QIVc, per age-group, based on estimates of relative vaccine effectiveness (rVE) between the two vaccines. VE estimates are taken from Rolfes et al. (16), and rVE from Boikos et al.(17), both studies were performed in the US during the 2017-2018 influenza season.

\begin{tabular}{|c|c|c|c|c|c|c|}
\hline & \multicolumn{3}{|c|}{ Match (16) } & Mismatch & Match & rVE(17) \\
\hline $\begin{array}{c}\text { Age } \\
\text { group }\end{array}$ & A/H1N1pdm09 & B Victoria & $\begin{array}{c}\mathrm{B} \\
\text { Yamagata }\end{array}$ & $\mathrm{A}(\mathrm{H} 3 \mathrm{~N} 2)$ & $\mathrm{A}(\mathrm{H} 3 \mathrm{~N} 2)$ & \\
\hline $\begin{array}{l}18- \\
49 \mathrm{yrs}\end{array}$ & $\begin{array}{c}48 \% \\
(18 \%-67 \%)\end{array}$ & $\begin{array}{c}57 \% \\
(43 \%-69 \%)\end{array}$ & $\begin{array}{c}57 \% \\
(43 \%-69 \%)\end{array}$ & $\begin{array}{c}14 \% \\
(0 \%-30 \%)\end{array}$ & $43 \%$ & $\begin{array}{c}26.8 \% \\
(14 \%-37 \%)\end{array}$ \\
\hline $\begin{array}{r}50- \\
64 \mathrm{yrs} \\
\end{array}$ & $36 \%(0 \%-67 \%)$ & $\begin{array}{c}44 \% \\
(24 \%-60 \%) \\
\end{array}$ & $\begin{array}{c}44 \% \\
(24 \%-60 \%) \\
\end{array}$ & $\begin{array}{c}21 \% \\
(0 \%-41) \\
\end{array}$ & $50 \%$ & \\
\hline
\end{tabular}

Note: We report 95\% confidence intervals (CI) in parenthesis. We have assumed a $0 \%$ in the lower bound $95 \%$ CI in vaccine effectiveness when negative percentage was reported by Rolfes et al (16). We used VE estimates against influenza B (all lineages), as age-specific estimates were not available by B lineages. 
Table 2 - Base case results -3 years of mismatch out of 5 years. The 3 years of mismatch considered are the 3 most recent influenza seasons.

\begin{tabular}{|c|c|c|c|}
\hline & QIVe & QIVc & Difference \\
\hline Number of doses & 118818800 & 118818800 & 0 \\
\hline Number of cases & 27240600 & 21556900 & -5683700 \\
\hline Number of GP* visits & 8595300 & 6751400 & -1843900 \\
\hline Lost work days & 29390500 & 22983500 & -6407000 \\
\hline Hospitalizations & 199916 & 150191 & -49725 \\
\hline Deaths & 22436 & 16983 & -5453 \\
\hline Life years lost & 371040 & 277635 & -93405 \\
\hline Life years lost (discounted) & 269745 & 202268 & -67477 \\
\hline QALY lost due to sickness & 165410 & 129151 & -36259 \\
\hline QALY lost due to death & 367118 & 274906 & -92212 \\
\hline $\begin{array}{l}\text { QALY lost due to death } \\
\text { (discounted) }\end{array}$ & 266001 & 199575 & -66426 \\
\hline Total QALY lost & 532527 & 404057 & -128470 \\
\hline Total QALY lost (discounted) & 431410 & 328726 & -102684 \\
\hline Cost of GP visits & $\begin{array}{c}1028822 \\
400\end{array}$ & 775428700 & -253393700 \\
\hline Cost of hospitalizations & $\begin{array}{c}3794189 \\
100\end{array}$ & 2775660700 & -1018528400 \\
\hline Cost of lost workdays & $\begin{array}{c}2105378 \\
600\end{array}$ & 1648894900 & -456483700 \\
\hline Vaccine cost & $\begin{array}{c}2610747 \\
400\end{array}$ & 3037887500 & 427140100 \\
\hline Total direct costs & $\begin{array}{c}7433759 \\
000\end{array}$ & 6588976900 & -844782100 \\
\hline ICER & & \multicolumn{2}{|c|}{$\begin{array}{l}-10400[-17400 ; 11000] \text { (cost } \\
\text { saving) }\end{array}$} \\
\hline
\end{tabular}

* General practitioner

Table 3: Sensitivity analysis on the number of mismatch years 


\begin{tabular}{|c|c|c|c|c|c|c|c|c|c|}
\hline & \multicolumn{3}{|c|}{ Medical costs* } & \multicolumn{3}{|c|}{ QALY** gained } & \multicolumn{3}{|c|}{ ICER*** } \\
\hline $\begin{array}{l}\text { Number of } \\
\text { mismatch years }\end{array}$ & Median & $\begin{array}{l}\text { Lower } \\
\text { bound }\end{array}$ & $\begin{array}{l}\text { Upper } \\
\text { bound }\end{array}$ & Median & $\begin{array}{l}\text { Lower } \\
\text { bound }\end{array}$ & $\begin{array}{l}\text { Upper } \\
\text { bound }\end{array}$ & Median & $\begin{array}{l}\text { Lower } \\
\text { bound }\end{array}$ & $\begin{array}{l}\text { Upper } \\
\text { bound }\end{array}$ \\
\hline 1 & $\begin{array}{c}-136179 \\
800\end{array}$ & $\begin{array}{c}-2176 \\
061100\end{array}$ & $\begin{array}{c}821374 \\
700\end{array}$ & 74800 & 10300 & $\begin{array}{l}205 \\
900\end{array}$ & -1800 & $\begin{array}{l}-12 \\
000\end{array}$ & $\begin{array}{c}67 \\
900\end{array}$ \\
\hline 2 & $\begin{array}{c}-1570 \\
682800\end{array}$ & $\begin{array}{c}-4916 \\
394100\end{array}$ & $\begin{array}{c}577624 \\
600\end{array}$ & $\begin{array}{l}164 \\
100\end{array}$ & 26800 & $\begin{array}{l}396 \\
300\end{array}$ & -8600 & $\begin{array}{l}-16 \\
000\end{array}$ & $\begin{array}{c}15 \\
000\end{array}$ \\
\hline 3 & $\begin{array}{c}-2749 \\
808800\end{array}$ & $\begin{array}{c}-7530 \\
202100\end{array}$ & $\begin{array}{c}351732 \\
800\end{array}$ & $\begin{array}{l}241 \\
200\end{array}$ & 42200 & $\begin{array}{l}600 \\
100\end{array}$ & -10500 & $\begin{array}{l}-18 \\
000\end{array}$ & 3400 \\
\hline 4 & $\begin{array}{c}-3999 \\
641400\end{array}$ & $\begin{array}{c}-9658 \\
482100\end{array}$ & $\begin{array}{c}182466 \\
200\end{array}$ & $\begin{array}{l}325 \\
700\end{array}$ & 50400 & $\begin{array}{l}732 \\
600\end{array}$ & -11600 & $\begin{array}{l}-18 \\
800\end{array}$ & 100 \\
\hline 5 & $\begin{array}{c}-5222 \\
697300\end{array}$ & $\begin{array}{l}-12426 \\
192400\end{array}$ & $\begin{array}{c}21380 \\
200\end{array}$ & $\begin{array}{l}412 \\
000\end{array}$ & 64600 & $\begin{array}{l}920 \\
200\end{array}$ & -12200 & $\begin{array}{l}-19 \\
400\end{array}$ & -3200 \\
\hline
\end{tabular}

* Medical costs include cost of GP visits and hospitalizations ** Quality-adjusted life year *** Incremental cost effectiveness ratio

\section{Figures}
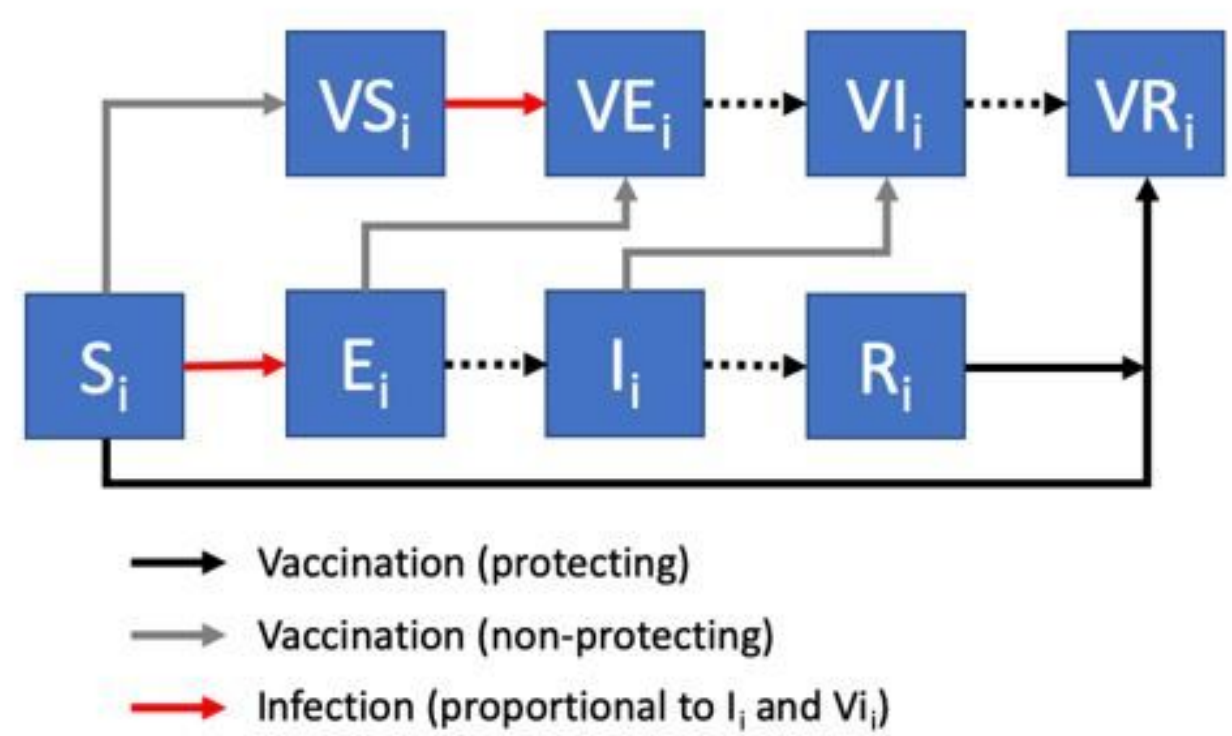

$\ldots$. State transition depending on natural history of influenza

\section{Figure 1}

Diagram of the influenza transmission model. Si, Ei, li, and Ri stand for susceptible, exposed, infectious, and recovered individuals respectively regarding the influenza strain I; VSi, VEi, and Vli stand for susceptible, exposed and infectious individuals experiencing a non-protective vaccination respectively regarding influenza strain $\mathrm{i}, \mathrm{VRi}$ are individuals protected and vaccinated against influenza strain ; $\mathrm{i}$ stands for influenza strains: A/H1N1, A/H3N2, B Victoria, B Yamagata 


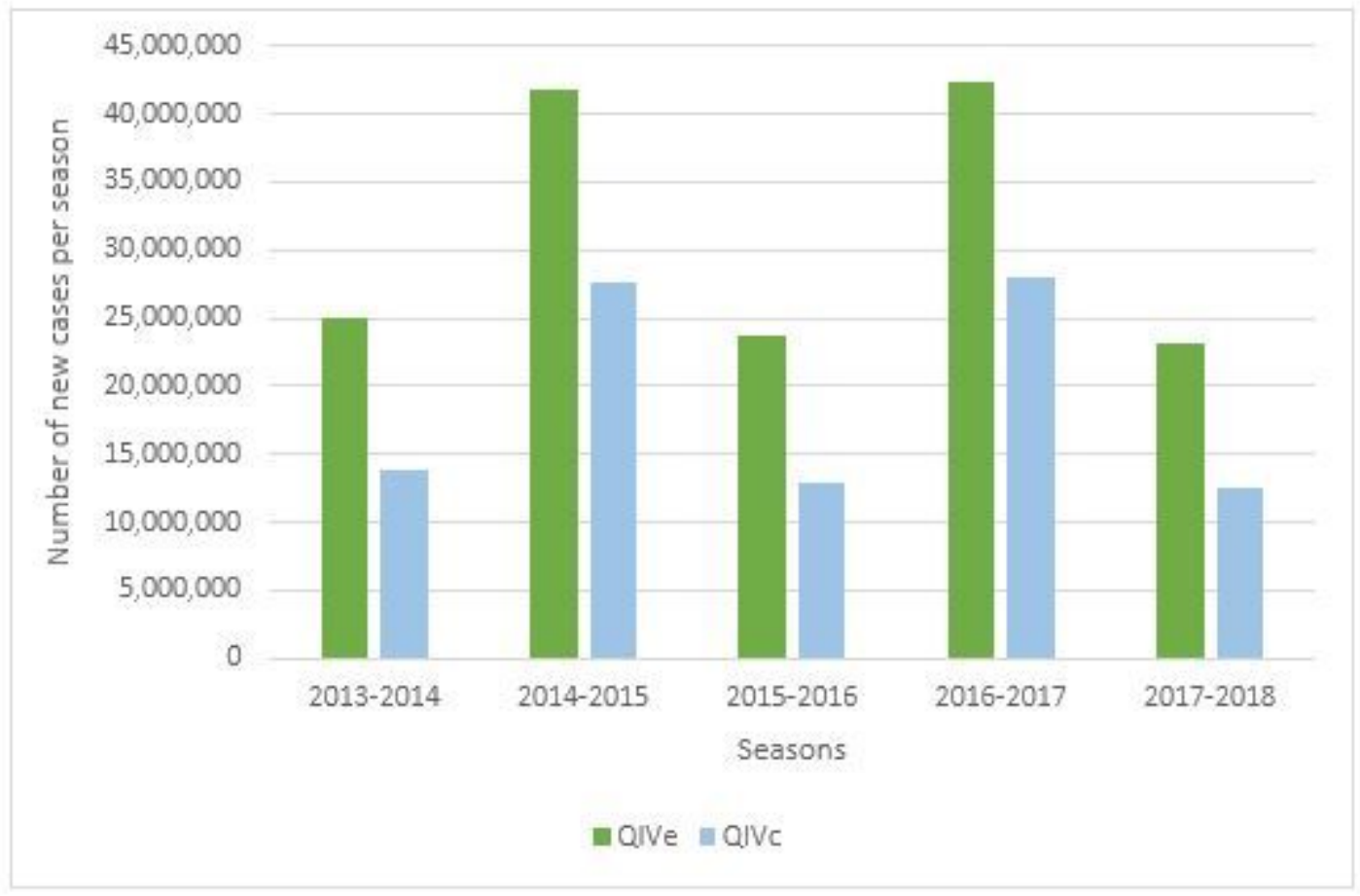

\section{Figure 2}

Number of influenza cases over the 5 studied influenza seasons considering systematic A(H3N2) mismatch

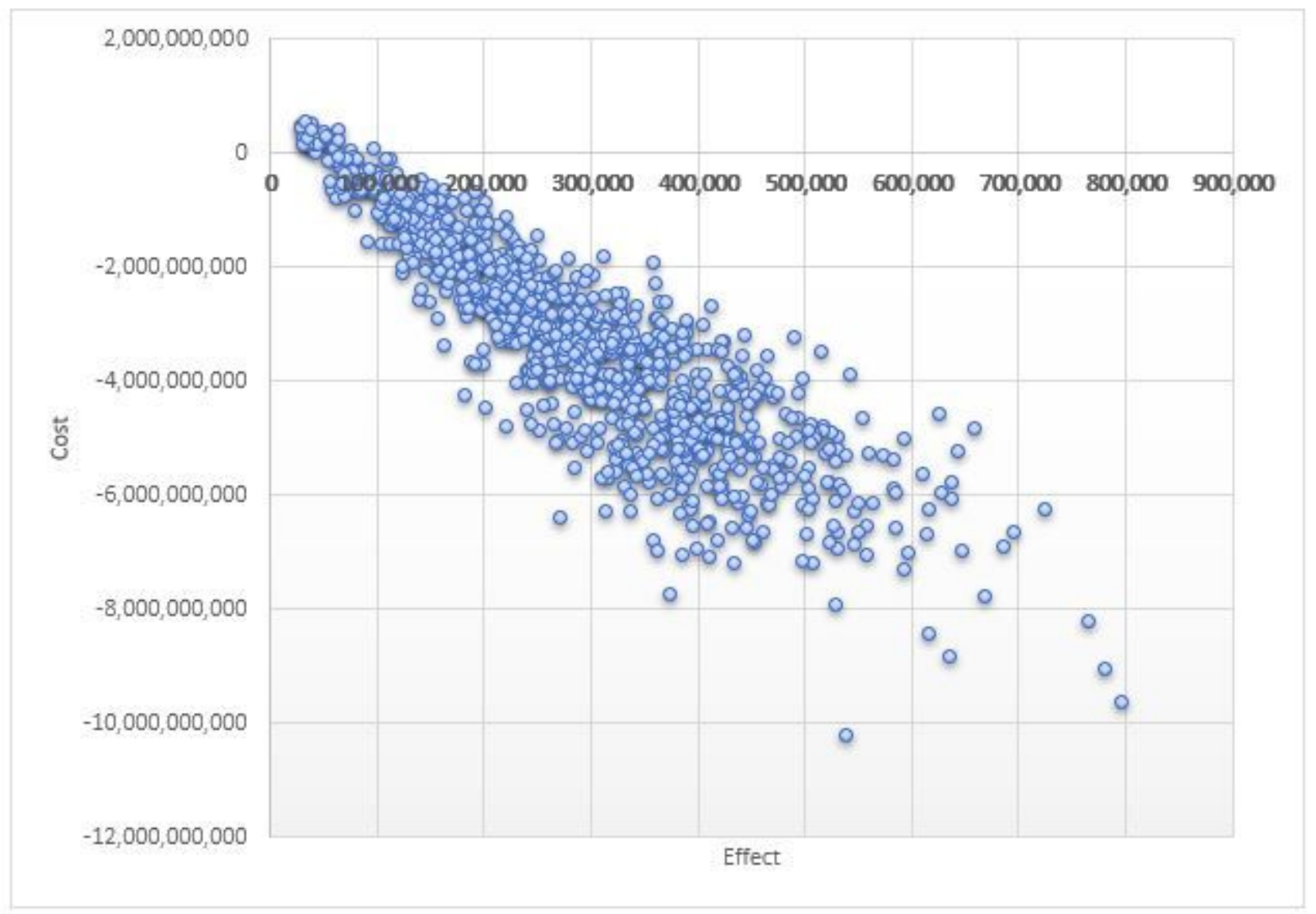


Figure 3

Probabilistic sensitivity analysis of the QIVc scenario compared to QIVe. Health is measured in QALY and cost in US\$. 